\title{
Observation of individual tracer atoms in an ultracold dilute gas
}

\author{
Michael Hohmann, ${ }^{1}$ Farina Kindermann, ${ }^{1}$ Tobias Lausch,${ }^{1}$ Daniel \\ Mayer, ${ }^{1,2}$ Felix Schmidt, ${ }^{1,2}$ Eric Lutz, ${ }^{3}$ and Artur Widera ${ }^{1,2}$ \\ ${ }^{1}$ Department of Physics and Research Center OPTIMAS, University of Kaiserslautern, Germany \\ ${ }^{2}$ Graduate School Materials Science in Mainz, Gottlieb-Daimler-Strasse 47, 67663 Kaiserslautern, Germany \\ ${ }^{3}$ Department of Physics, Friedrich-Alexander-Universität Erlangen-Nürnberg, 91058 Erlangen, Germany
}

(Dated: July 16, 2018)

\begin{abstract}
Understanding the motion of a tracer particle in a rarefied gas is of fundamental and practical importance. We report the experimental investigation of individual Cs atoms impinging on a dilute cloud of ultracold $\mathrm{Rb}$ atoms with variable density. We study the nonequilibrium relaxation of the initial nonthermal state and detect the effect of single collisions which has eluded observation so far. We show that after few collisions, the measured spatial distribution of the light tracer atoms is correctly described by a generalized Langevin equation with a velocity-dependent friction coefficient, over a large range of Knudsen numbers.
\end{abstract}

Diffusion is an essential and omnipresent transport phenomenon in nature. The motion of a tagged particle in a fluid is determined by its mass $m$ and by the density of the fluid via the Knudsen number $K_{n}$ [1]. In the regime of large densities $\left(K_{n} \ll 1\right)$, the interparticle collision frequency is high and the fluid may be treated as a continuum medium. On the other hand, for low densities $\left(K_{n} \gg 1\right)$, individual collisions matter and the discrete nature of the fluid is apparent. The only closed equation applicable to all values of $K_{n}$ is the Boltzmann equation for the phase-space distribution of the particles [1, 2. Solutions of this nonlinear kinetic equation have been obtained in the extreme situations of vanishing and infinite Knudsen numbers, and in the Brownian limit of a heavy tracer particle, $m / M \gg 1$, where $M$ is the mass of the fluid particles 1, 2, However, despite its central importance for the foundations of statistical physics and the study of e.g. fluid flows in the upper atmosphere and aerosols dynamics 2 4, much less is known, both theoretically and experimentally, about the long-standing problem of light tagged particles diffusing in a dilute gas at intermediate $K_{n}[\underline{5}$.

An alternative and highly successful description of a tagged particle is offered by the Langevin equation [6, 7]. In this stochastic approach, Newton's equation of motion for the particle is extended by a friction force and a fluctuating force that account for the interaction with the surrounding fluid. The Langevin equation enables simple evaluation of the macroscopic properties of the diffusing particle, without the need to compute complicated collision integrals as in the Boltzmann equation. It is valid in the Brownian limit of a heavy tracer particle, where the friction coefficient is independent of the velocity of the particle [8]. Recent experimental studies of Brownian motion have been reported in gases [9, 10] and liquids [11, 12. However, the usual Langevin equation with constant friction does not hold for light tracer particles in dilute gases [13, 14].

Here, we experimentally observe the motion of individual ${ }^{133} \mathrm{Cs}$ atoms impinging on a dilute cloud of ultracold ${ }^{87} \mathrm{Rb}$ atoms as shown in Fig. 1. We exploit the variable density of the cloud to explore a wide range of Knudsen numbers, from $K_{n} \simeq 1$ at the center to arbitrarily large values at the edges, for the light Cs tracer atom with $m / M \simeq 1.5$. By initially accelerating the laser cooled Cs atoms to a nonthermal kinetic energy of about $43 k T$, we are able to investigate in detail the nonequilibrium relaxation induced by the collisions with the cloud particles, from ballistic to diffusive motion ( $T$ is here the temperature of the thermal $\mathrm{Rb}$ cloud and $k$ the Boltzmann constant). The high resolution of our setup further allows to detect the effect of a single collision on the dynamics of the tracer atom. Discrete hard-sphere collision simulations reveal that only few collisions suffice to thermalize a Cs atom to the cloud temperature. We additionally demonstrate that the measured spatial distribution of tagged Cs atoms is well described, without any free parameters, by a generalized Langevin equation with a velocity-dependent friction coefficient [13, 14]. To our knowledge, this extension of the Langevin equation has never been experimentally verified so far.

In our experiment, laser cooled ${ }^{133} \mathrm{Cs}$ atoms are released from a magneto-optical trap (MOT) into a crossed dipole trap containing a dilute gas of ${ }^{87} \mathrm{Rb}$ atoms (Fig. 1 (a,b,c)) [15. After entering the cloud, a Cs atom undergoes collisions with the cold Rb atoms and thermalizes. Ultracold temperatures in the micro Kelvin range lead to slow dynamics, both for the gas and the tracer atoms, with thermal velocities around $10 \mathrm{~mm} \mathrm{~s}^{-1}$. While at room temperature, heavy tracer particles are bombarded at an extremely high rate (of the order of $10^{16}$ $\mathrm{Hz}$ in air [16]), ultra low temperatures, a small mass ratio of the order of unity and low gas densities reduce this collision rate to values between $13 \times 10^{3} \mathrm{~Hz}$ at the center and 0 at the edges of the gas cloud. Hence the effective mean free time between two collisions is in the experimentally accessible range of $\geq 0.1 \mathrm{~ms}$. For the finite size cloud, there is a non-zero probability that a Cs atom does not collide at all and moves unperturbed through the cloud (Fig. 1(f)). In order to observe the spatial distribution of the tagged Cs atoms, we freeze their positions after a given (interaction) time $t$ after release from 

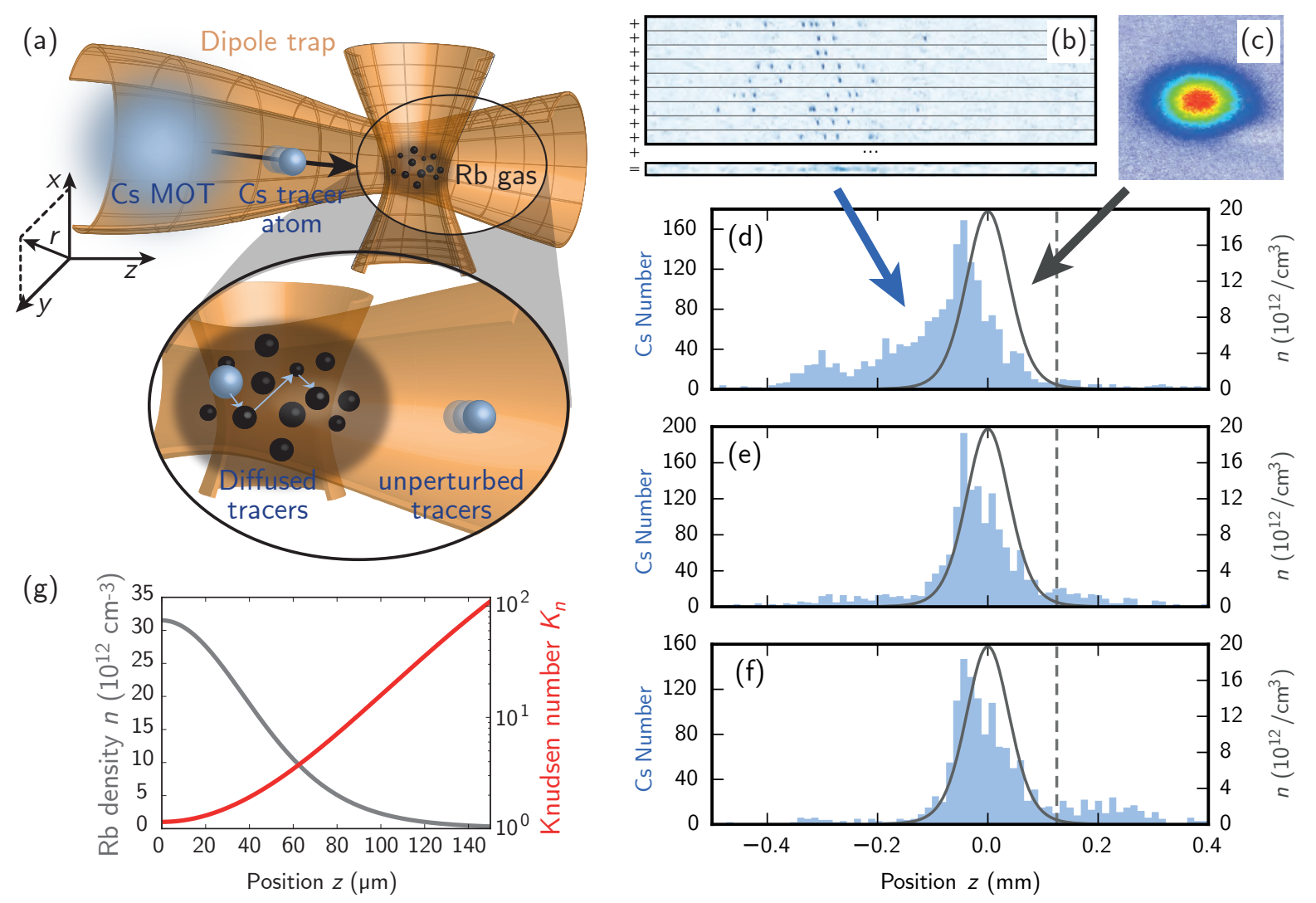

FIG. 1. Sketch of experimental sequence. (a) Individual Cs tracer atoms are released from a MOT at $z=-0.27 \mathrm{~mm}$ and are drawn towards the center of a crossed dipole trap containing an ultracold thermal Rb cloud, where all atoms are in their respective hyperfine ground state. The Rb cloud contains typically $6 \times 10^{3}$ to $3.5 \times 10^{4}$ atoms at a temperature of $2 \mu \mathrm{K}$ and with $1 / e^{2}$ widths of $\sigma_{r}=1.3 \mu \mathrm{m}$ and $\sigma_{z}=39 \mu \mathrm{m}$ in radial and axial directions. (b) After a variable (interaction) time $t$ after tracer release, the atomic motion is frozen by turning on a strong optical lattice. The position of individual Cs atoms is detected by fluorescence imaging after the $\mathrm{Rb}$ cloud has been removed. A series of fluorescence images for identical experimental conditions yields the spatial distribution of tracer atoms. (c) Time-of-flight image of the dilute Rb cloud. (d-f) Measured spatial tracer distribution for a central $\mathrm{Rb}$ density of $n=2 \times 10^{13} \mathrm{~cm}^{-3}$ for increasing interaction time $t$ : (d) the Cs tracer distribution impinges onto the Rb cloud at $17 \mathrm{~ms}$; (e) the Cs tracer distribution is axially overlapped with the Rb cloud at $21 \mathrm{~ms}$; and (f) tracers which have not collided have left the region of the Rb cloud at $25 \mathrm{~ms}$. The grey Gaussian curve indicates the Rb cloud density, right vertical scale applies. The dashed vertical line at $z=0.125 \mathrm{~mm}$ separates the diffused fraction that has thermalized inside the cloud (left) from the unperturbed fraction that has passed through the cloud (right). (g) Cloud density profile and corresponding Knudsen number variation for the highest central density of $n=3.2 \times 10^{13} \mathrm{~cm}^{-3} \mathrm{considered}$.

the MOT by turning on a strong $1 \mathrm{D}$ optical lattice in the $z$-direction. We subsequently remove the $\mathrm{Rb}$ cloud from the trap and record the atomic position with fluorescence imaging (Fig. 11(b)) [17, 18. The spatial distribution for any fixed time is determined by accumulating atomic positions over about 600 realizations. The time evolution of the distribution is obtained by varying the interaction time $t$ (Figs. 1](d)-(f)). We use only a few Cs atoms $(\approx 7$ on average) per experimental run. Interspecies effects such as self-diffusion and self-thermalization are therefore negligible. Moreover, the thermal de Broglie wavelength is smaller than the interparticle distance for the densities considered [19] and the dynamics of the atoms can hence be described classically.

Figures 2(a)-(c) show the measured Cs spatial distri- butions for three increasing values of the peak $\mathrm{Rb}$ density $n_{\text {max }}$ after an interaction time $t=25 \mathrm{~ms}$. For low Rb density (Fig. 2(a)), we observe a bimodal distribution with a fraction of unperturbed Cs atoms (right) that has passed through the Rb cloud (grey Gaussian curve) and a larger fraction of diffused atoms that has thermalized inside the cloud (left). The existence of the unperturbed fraction reveals the discrete nature of the dilute $\mathrm{Rb}$ gas. When the Rb density is increased (Figs. 2(b)-(c)), the unperturbed fraction shrinks, as the collision probability grows with $n_{\max }$, and the diffused fraction does not fully penetrate the cloud. This may be qualitatively understood by noting that diffusion slows down with increasing density so that Cs tracer atoms need more time to reach the center of the cloud. In addition, three-body losses become im- 

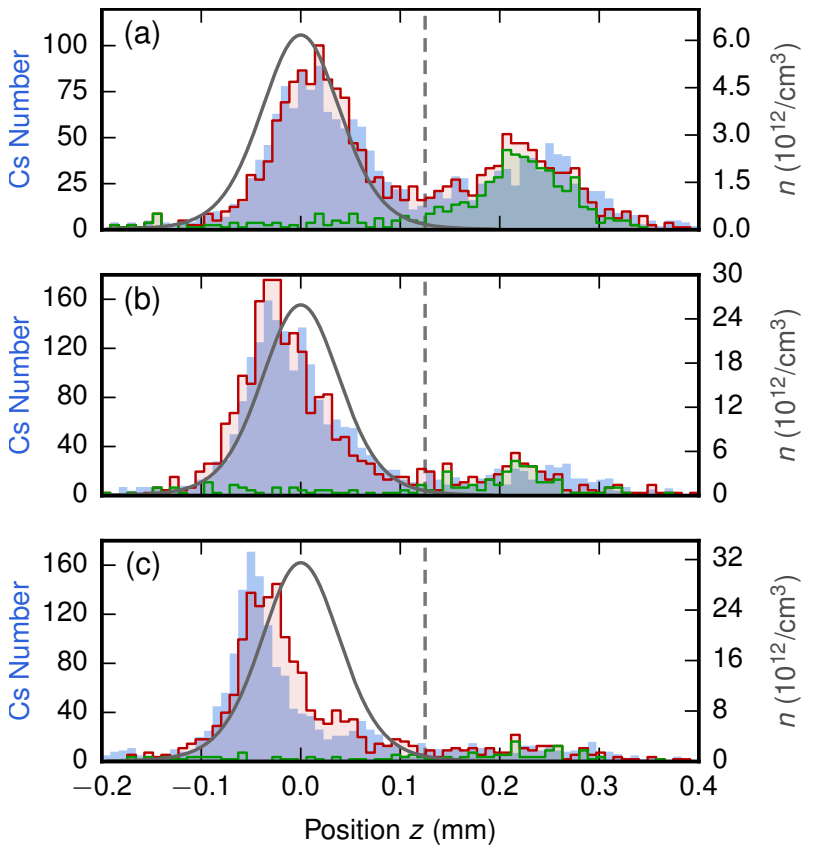

FIG. 2. Spatial distribution of Cs tracer atoms. Measured (blue shaded) and collision simulated (red line) spatial distributions after an interaction time of $25 \mathrm{~ms}$ for Rb center densities of (a) $n=6 \times 10^{12} \mathrm{~cm}^{-3}$, (b) $n=2.6 \times 10^{13} \mathrm{~cm}^{-3}$, and (c) $n=3.2 \times 10^{13} \mathrm{~cm}^{-3}$. The simulations are performed with 1500 atoms and normalized to the measured distribution. The agreement is excellent for both diffused (left) and unperturbed (right) fractions with an overlap larger than $90 \%$. The unperturbed fraction is mostly composed of tracer atoms that have not suffered any collision (green, simulated).

portant close to the center, where the density is highest, further decreasing the number of Cs atoms. A measurement of the three-body loss rate is presented in Ref. 19]. The lifetime of a Cs atom at the peak of the spatial distribution in Fig. 2(c) is $\tau=3 \mathrm{~ms}$. Three-body-losses are therefore accounted for in all simulations.

In order to quantitatively describe the measured spatial distribution and the thermalization process, we first employ discrete hard-sphere collision simulations [19. We numerically solve Newton's equation of motion for individual Cs tracer atoms elastically colliding with the thermal gas of $\mathrm{Rb}$ atoms in three dimensions. Spatial distributions as shown in Fig. 2 were obtained by projecting the atomic positions onto the $z$-axis. A tagged Cs atom initially starts with an effective Gaussian spatial distribution at $z=-0.27 \mathrm{~mm}$ with width $\sigma_{x}=$ $\sigma_{y}=6.8 \mu \mathrm{m}$ and $\sigma_{z}=58 \mu \mathrm{m}$ at time $t=7 \mathrm{~ms}$, and a Maxwell-Boltzmann velocity distribution with temperature $1.4 \mu \mathrm{K}$. These parameters were extracted from an independent time-resolved reference measurement in the absence of the cloud. The properties of the $\mathrm{Rb}$ cloud were also obtained by independent measurements, so that there are no free parameters in the simulations. At each time step $d t$, a collision between a tracer atom and a
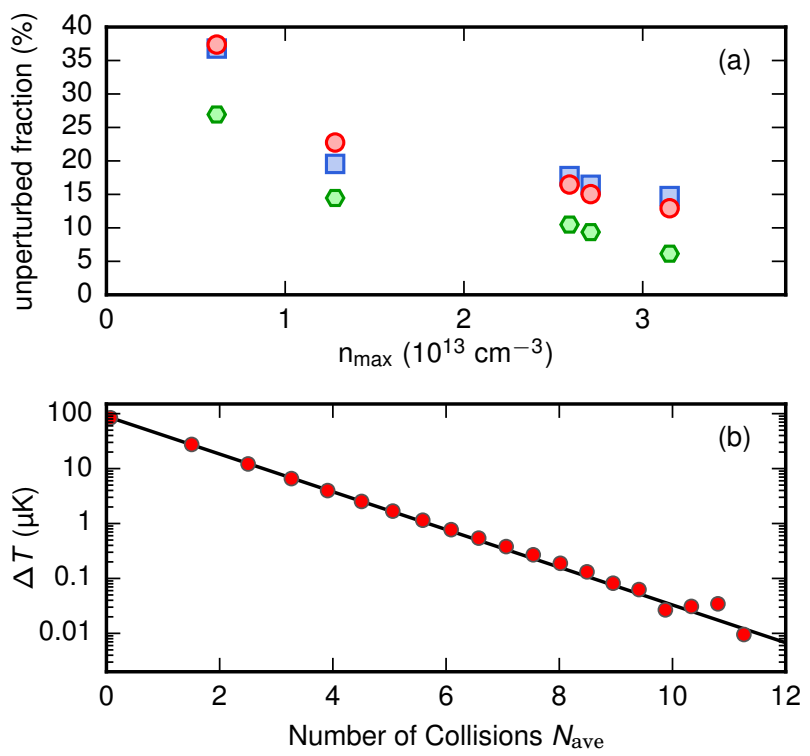

FIG. 3. Collision free atoms and thermalization. (a) Measured (blue squares) and collision simulated (red dots) relative number of atoms in the unperturbed fraction $(z>$ $0.125 \mathrm{~mm}$ ) for various maximum $\mathrm{Rb}$ densities $n_{\max }$. Good agreement with simulated collision free Cs atoms (green hexagons) is observed. (b) Simulated exponential decay of the difference $\Delta T$ between the Cs kinetic temperature and the $\mathrm{Rb}$ temperature, as a function of the average number of collisions $N_{\text {ave }}$ in a homogeneous cloud. The solid line is an exponential fit to the data with a $1 / e$ number of collisions of 1.3 .

$\mathrm{Rb}$ atom is described by a local collision probability, $P_{\text {coll }}=1-\exp (-\Gamma d t)$, where $\Gamma$ is the collision rate. For nonthermal test particles with velocity $v$, the collision rate is given by (Eq. 5.4,5 in Ref. [20])

$$
\Gamma(v)=n \frac{\sigma}{\pi} \sqrt{\frac{2 \pi k T}{M}}\left[e^{-x^{2}}+\left(2 x+\frac{1}{x}\right) \frac{\sqrt{\pi}}{2} \operatorname{Erf}(x)\right],
$$

where $\sigma$ is the scattering cross section, $x^{2}=M v^{2} /(2 k T)$ and $\operatorname{Erf}(x)$ the error function. We analogously describe three-body losses by the probability $P_{\text {loss }}=1-$ $\exp (-d t / \tau)$, with the local lifetime $\tau$ [19. Since the density $n$ changes spatially, the expressions above are evaluated at the current position of a tracer.

The results of the discrete collision simulations are compared to experimental tracer distributions in Figs. 2(a)-(c). We obtain excellent agreement between measured (blue) and simulated (red) spatial distributions, both for the unperturbed (right) and the diffused fraction (left), with an overlap, $\left(\int N_{\exp } N_{\text {sim }} d z\right) /\left(\int N_{\exp }^{2} d z \int N_{\text {sim }}^{2} d z\right)^{\frac{1}{2}}$, between the two distributions of more than $90 \%$. We attribute the slight discrepancies to measurement uncertainties of the independently determined parameters entering the simulations [19. Figure 3(a) depicts the relative number of atoms in the unperturbed fraction (defined as those 

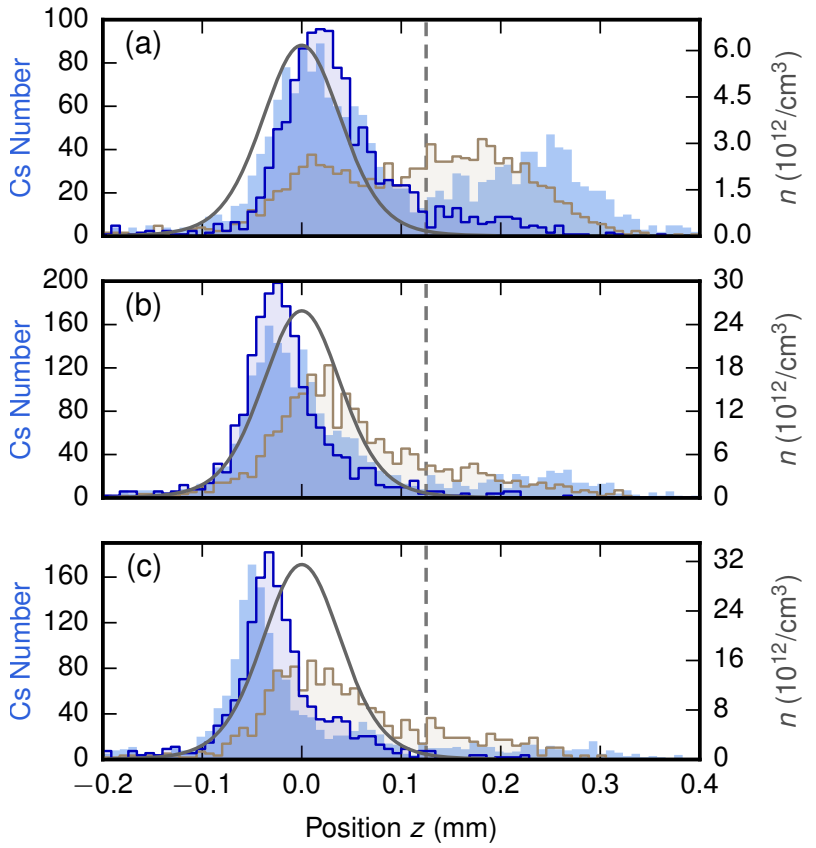

FIG. 4. Spatial distribution of Cs atoms - Langevin description. Measured (blue shaded) and the Langevin simulated spatial distribution (blue line for velocity-dependent friction, brown line for velocity-independent friction [19]) for the same data as in Fig. 2 The simulations are performed with 1500 atoms and normalized to the diffused fraction. Excellent agreement is found for the thermalized diffused fraction (left) between measured data and velocity dependent friction Langevin description, with overlap larger than $90 \%$. By contrast, the velocity-independent description yields only poor agreement with measured data with overlaps as low as $60 \%$. In both cases, the Langevin equation (2) does not capture the unperturbed fraction (right), as it assumes that all tracer atoms experience damping and noise.

atoms with position $z>0.125 \mathrm{~mm}$, marked by the vertical dashed line), for various values of the maximum Rb density $n_{\max }$. The simulations show that the unperturbed fraction is mostly composed of tracer atoms that do not undergo any collision (green, simulated). The small difference between the unperturbed and noncollided fractions is due to the sharp cut at $z=0.125 \mathrm{~mm}$. In addition, we find that the computed temperature difference $\Delta T$ between the kinetic temperature of the tagged Cs atoms and the cloud temperature decays exponentially with the number of average collisions $N_{\text {ave }}$ with a 1/e decay constant of 1.3 collisions (Fig. 3 (b)). This indicates thermalization after a few collisions. We estimate, for example, a relative temperature difference of $\Delta T / T \simeq 2 \%$ after $4 \times 1.3=5.2$ collisions.

We next test the validity of the stochastic Langevin approach by simulating single particle Langevin trajectories [6]. For discrete time steps $d t$, the Langevin equation for the velocity $\vec{v}$ of a tagged particle takes the form [7]

$$
\vec{v}(t+d t)=\vec{v}(t)+\left(\vec{F}_{\text {drag }}+\vec{F}_{\text {rand }}-\vec{\nabla} U\right) \frac{d t}{m}
$$

Here, $\vec{F}_{\mathrm{drag}}=-\gamma \vec{v}$ is a drag force with friction coefficient $\gamma, \vec{F}_{\text {rand }}$ a fluctuating force and $-\vec{\nabla} U$ the confining force including gravity. While for large, heavy test particles the friction coefficient $\gamma$ is velocity independent, it acquires an explicit velocity dependence for small, light particles [13, 14]. It was theoretically shown that [13, 14]

$$
\gamma(v)=n \sigma \frac{8}{15} \sqrt{\frac{2 M}{\pi k T}} \frac{m\left(M v^{2} / 2+5 k T\right)}{(m+M)}
$$

to order $(M / m)^{3 / 2}$ and for values of $m v^{2} /(2 k T)$ not much larger than unity. To our knowledge, the predictions of the Langevin equation with speed-dependent damping coefficient (3) have never been verified experimentally so far. We also note that owing to the detailed-balance condition, the nonlinear friction (3) implies a multiplicative, velocity-dependent fluctuating force [19, 21.

The results of the Langevin simulations are presented in Fig. 4 [19. We have again accounted for three-body losses by removing tracer atoms at each time step with the probability $P_{\text {loss }}$. As before, the cloud density $n$, and thus the friction coefficient $\gamma$, were evaluated at the position of the tagged atoms. We observe that the diffused fraction of Cs atoms (left) is well described by the Langevin simulations (blue), without free parameters, for all values of the Rb density. However, as expected, the unperturbed fraction (right) is not captured by the Langevin approach which assumes that all test particles experience damping and fluctuations. The overlap between the simulated and measured spatial distributions for the diffused fraction is larger than $90 \%$. We once more ascribe the small deviation to the experimental uncertainties of the reference measurements. By contrast, Langevin simulations with the usual velocityindependent friction coefficient (brown) yield overlaps as low as $60 \%$ 19.

The following physical picture emerges from our experimental and theoretical investigations. Our ability to accurately identify the effect of a single collision allows the distinction of two different types of dynamics: Thus, Cs atoms that do not experience any collision pass ballistically through the dilute Rb cloud (unperturbed fraction). On the other hand, a single collision event dissipates on average more than half of the initial nonthermal kinetic energy and suffices to trap a tagged Cs atom inside the cloud, leading to additional collisions (diffused fraction). After few more collisions, a tracer atom will be thermalized with the Rb cloud. Its dynamics is correctly described, over a wide range of Knudsen numbers, by a generalized Langevin equation with a velocity-dependent friction coefficient. The origin of this unfamiliar speed dependence may be understood by noting that the velocity $v$ of a heavy Brownian particle $(m \gg M)$ is much smaller than that of the gas atoms. As a result, both 
the collision rate and the friction coefficient are independent of $v$ in this limit. By contrast, for an atomic tracer with a mass $m \simeq M$, both velocities are of the same order. Here, an explicit velocity dependence of the collision rate and of the friction coefficient can no longer be neglected. However, as we have shown, in both cases, a highly successful continuous Langevin description over a coarse-grained timescale larger than the mean free time between impacts is possible.

We thank Axel Pelster and James Anglin for helpful discussions. This work was partially funded by the ERC Starting Grant Nr. 278208, the Collaborative Project TherMiQ (Grant Agreement 618074) and the SFB/TRR49. T. L. acknowledges funding by Carl Zeiss Stiftung, D.M. is a recipient of a DFG-fellowship through the Excellence Initiative by the Graduate School Materials Science in Mainz (GSC 266), F.S. acknowledges funding by the Studienstiftung des deutschen Volkes.

\section{SUPPLEMENTARY MATERIAL}

\section{A. Knudsen number}

The Knudsen number is defined as $K_{n}=\lambda / L$, where $\lambda$ is the density-dependent mean-free path within the gas and $L$ is the typical length scale associated with the diffusing particle [1]. For the Cs atoms, the mean free path is given by $\lambda=1 /(\sqrt{2} n \sigma)$, where $\sigma=4 \pi a_{\mathrm{Rb}, \mathrm{Cs}}^{2}$ is the interspecies scattering cross-section and $a_{\mathrm{Rb}, \mathrm{Cs}}=$ $645 a_{B} 22$ the $s$-wave scattering length between two $\mathrm{Rb}$ atoms ( $a_{B}$ is the Bohr radius). On the other hand, in the absence of the $\mathrm{Rb}$ cloud, the dynamics is dominated by collisions with the trapping potential in the radial directions $(x, y)$. We thus identify $L=\sqrt{k T /\left(M \omega_{r}^{2}\right)}$, the radial $1 / e$ width of the thermal $\mathrm{Rb}$ cloud with $\omega_{r}$ the radial angular trap frequency.

\section{B. Negligible quantum effects}

Despite using ultracold temperatures in the micro Kelvin range, the dynamics of the atoms in our experiments can be described classically. Quantum effects are in general relevant when the condition $\lambda_{\mathrm{th}}^{3} / n \leq 1$, with $\lambda_{\text {th }}$ the thermal de Broglie wavelength, is satified [23]. For the highest $\mathrm{Rb}$ density measured, we have instead $\lambda_{\mathrm{th}, \mathrm{Rb}}^{3} / n=16$ for $\mathrm{Rb}$ and $\lambda_{\mathrm{th}, \mathrm{Cs}}^{3} / n=30$ for Cs. In addition, the mean-field contribution of the cold Rb cloud to the effective potential on Cs [23],

$$
V_{\mathrm{mf}}=n g_{\mathrm{RbCs}}=n \frac{4 \pi \hbar^{2} a_{\mathrm{RbCs}}}{\mu},
$$

with $\mu$ the reduced mass, can be used as an order-ofmagnitude estimate for the highest $\mathrm{Rb}$ density. We have $V_{\mathrm{mf}} \simeq k \times 0.1 \mu \mathrm{K}$, a value which is negligible compared to the potential depth of $k \times 270 \mu \mathrm{K}$ for Cs and the bath's

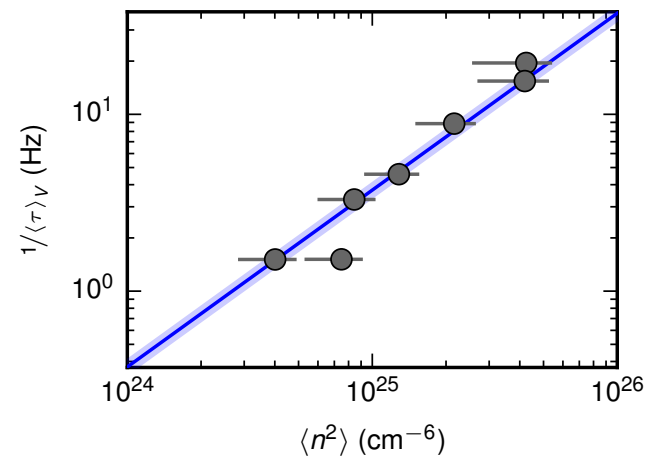

FIG. 5. Measurement of the three-body loss coefficient $L_{3}$ for $\mathrm{Cs}$ in $|F=3\rangle$ and $\mathrm{Rb}$ in $\left|F=1, m_{F}=0\right\rangle$ Circles: Measured lifetimes. Blue solid line: Fit of Eq. (5) to the data yielding $L_{3}=37(3) 10^{-26} \mathrm{~cm}^{6} \mathrm{~s}^{-1}$. The error marks the $1 \sigma$ confidence interval originating from statistical uncertainties and is visualized by the blue shaded area.

thermal energy scale $k T=k \times 2 \mu \mathrm{K}$. Furthermore, the dynamics of the Cs atoms in the cloud are not dominated by the trapping potential, but by the Rb density distribution which experiences a six times lower mean field shift due to the lower Rb-Rb scattering length [24].

\section{Three-body loss rate}

The lifetime of Cs atoms in our experiment is limited by three-body losses. This influences the evolution of the Cs atom's spatial distribution. Therefore, we have measured the inverse three-body loss rate as a function of $\mathrm{Rb}$ density and account for three-body loss in our simulations. The spatially averaged lifetime is 23 ,

$$
\frac{1}{\langle\tau\rangle_{V}}=L_{3}\left\langle n_{\mathrm{Cs}} n^{2}\right\rangle_{V}
$$

where $L_{3}$ is the three-body loss coefficient and $\langle\cdot\rangle_{V}$ denotes the spatial average over the cloud's volume $V$. The local lifetime of a Cs atom is proportional to $n^{2}$, so Cs loss predominantly happens at the center of the Rb cloud. To determine the coefficient $L_{3}$, we measure $\langle\tau\rangle_{V}$ for various Rb densities by inserting Cs atoms into the cold cloud and recording the spatial distribution at various interaction times. Fitting Eq. (5) to the experimental data, as shown in Fig. 5 yields $L_{3}=37(3) 10^{-26} \mathrm{~cm}^{6} \mathrm{~s}^{-1}$. Here, $\mathrm{Cs}$ is prepared in $|F=3\rangle$ and $\mathrm{Rb}$ in $\left|F=1, m_{F}=0\right\rangle$ and the loss coefficient is roughly one order of magnitude larger than for $\mathrm{Cs}$ in the $\left|F=3, m_{F}=3\right\rangle$ and $\mathrm{Rb}$ in the $\left|F=1, m_{F}=1\right\rangle$ state [25]. Using the local lifetime $\tau(n)$ as a function of density, allows accounting for three-body recombination in our simulations. 


\section{Discrete collision simulations}

In this section, we present details of the hard-sphere collision simulations. We describe Cs tracer atoms as point-like masses that evolve in the absence of a collision according to Newton's equation of motion, $\vec{v}(t+d t)=$ $\vec{v}(t)-\vec{\nabla} U d t / m$, during each time step $d t$, where $U$ is the confining potential including gravity. We model the Rb cloud as a thermalized classical gas with timeindependent density $n$ and temperature $T$. This assumption is justified in view of the extreme number ratio between $\mathrm{Cs}$ and $\mathrm{Rb}$ atoms. At each time step, the local collision probability $P_{\text {coll }}=1-\exp (-\Gamma d t)$ with the position and velocity dependent collision rate $\Gamma$ is computed. Comparing $P_{\text {coll }}$ to a uniform random number determines if the collision occurs. For each collision event, a Rb collision partner is randomly generated and the Cs atom is assigned a new velocity $v$ by solving the two-body collision in the center-of-mass system under the assumption of isotropic $s$-wave scattering. This assumption is compatible with our hard-sphere model and well justified for the kinetic energies involved. The Rb atom does not play any further role in the simulation as a new, statistically representative $\mathrm{Rb}$ atom is drawn for each collision. Such a procedure is justified because the gas is dilute and correlations due to multiple scatterings with the same $R b$ atom can be neglected. We account for three-body losses with the probability $P_{\text {loss }}=1-\exp (-d t / \tau)$, with the local lifetime $\tau$. In view of the initial nonthermal kinetic energy of the tagged atoms, the velocity $\mathbf{V}$ of the $\mathrm{Rb}$ collision particle before the collision should be determined with care. Drawing the velocity of the $\mathrm{Rb}$ atom from a Maxwell-Boltzmann distribution is flawed, as the speeddependence of $\Gamma$ would depopulate high-velocity classes of $v$ due to more frequent scattering and prevent thermalization. This is the same reason, why the fluctuating force in the Langevin equation becomes speed dependent. The $\mathrm{Rb}$ velocity should instead be drawn from a modified (not normalized) Maxwell-Boltzmann distribution given by (see Ref. [20], Chapter 5.4),

$$
\begin{aligned}
P_{V}(v) & =\pi \int_{0}^{\pi} g(V, v, \theta) \sin \theta d \theta \int_{0}^{2 \pi} d \phi \frac{\sigma}{\pi} f_{M} V^{2} \\
P_{\theta}(v, V) & =g(V, v, \theta) \sin \theta \\
P_{\phi} & =1
\end{aligned}
$$

where $V, \theta$ and $\phi$ are polar coordinates of the Rb speed vector about $\mathbf{v}$ as an axis with probability distributions $P_{V}, P_{\theta}, P_{\phi} ; g$ is the relative velocity of the two collision partners and $f_{M}$ the usual Maxwell-Boltzmanndistribution

$$
f_{M}=n\left(\frac{M}{2 \pi k T}\right)^{\frac{3}{2}} \exp \left(\frac{-M V^{2}}{2 k T}\right) .
$$
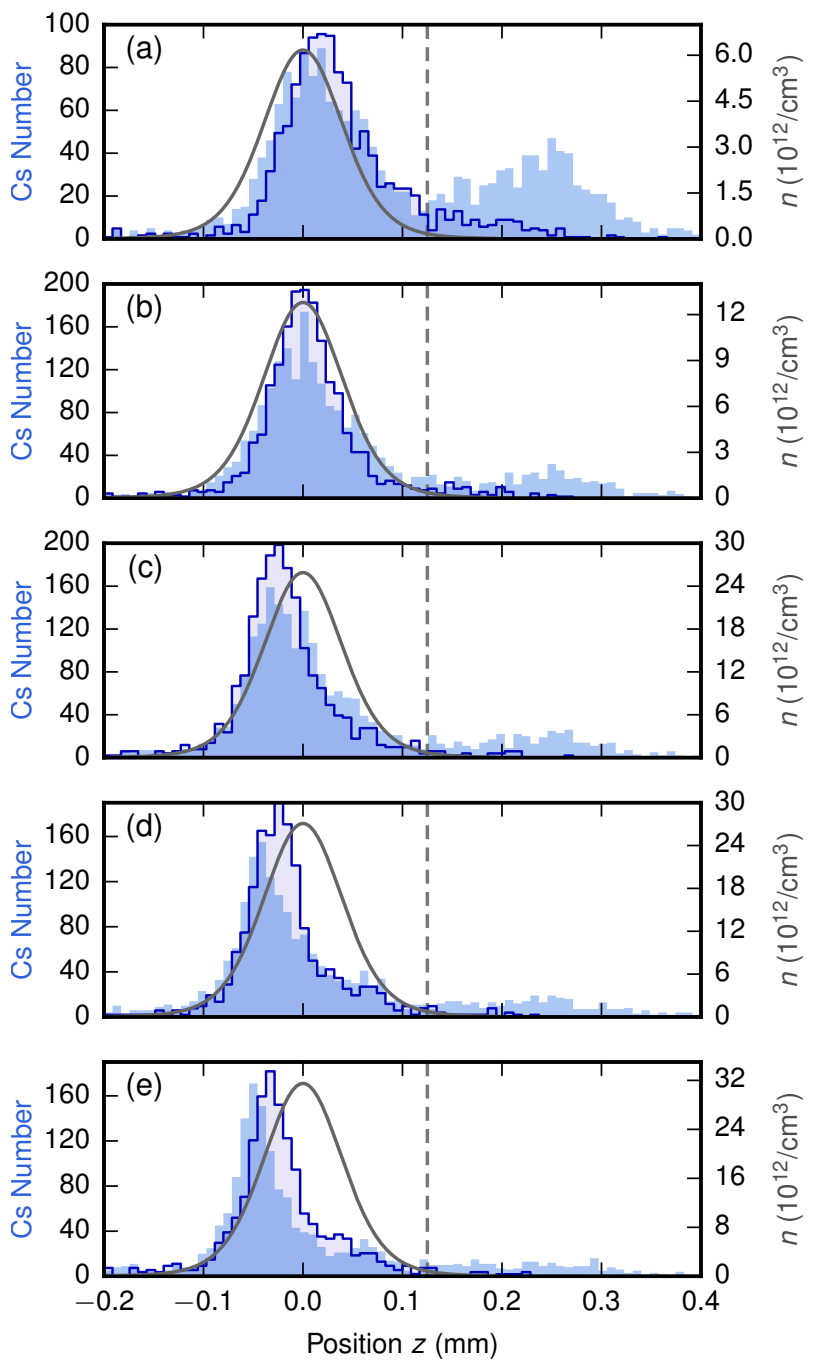

FIG. 6. Measured Cs distributions (blue histograms, borderless) for various peak $\mathrm{Rb}$ densities at $t=25 \mathrm{~ms}$ with Langevin simulated distributions (blue histogram, solid border), normalized to the diffused fraction.

\section{E. Langevin simulations}

We numerically solve the Langevin equation using a 3rd-order Runge-Kutta integrator with a typical time step of $d t=2 \mu \mathrm{s}$ and 1500 trajectories, corresponding to the number of observed Cs atoms. The fluctuationdissipation relation for an equilibrium bath implies a direct connection between damping and noise terms. For the velocity-dependent friction coefficient,

$$
\gamma(v)=n \sigma \frac{8}{15} \sqrt{\frac{2 M}{\pi k T}} \frac{m\left(M v^{2} / 2+5 k T\right)}{(m+M)}
$$

the detailed-balance condition and the Gibbs form of the thermal equilibrium condition lead to the speeddependent random force $\vec{F}_{\text {rand }}=\psi(v) \sqrt{2 / d t} \vec{N}$, where $\vec{N}$ is a normally distributed random force with zero mean 

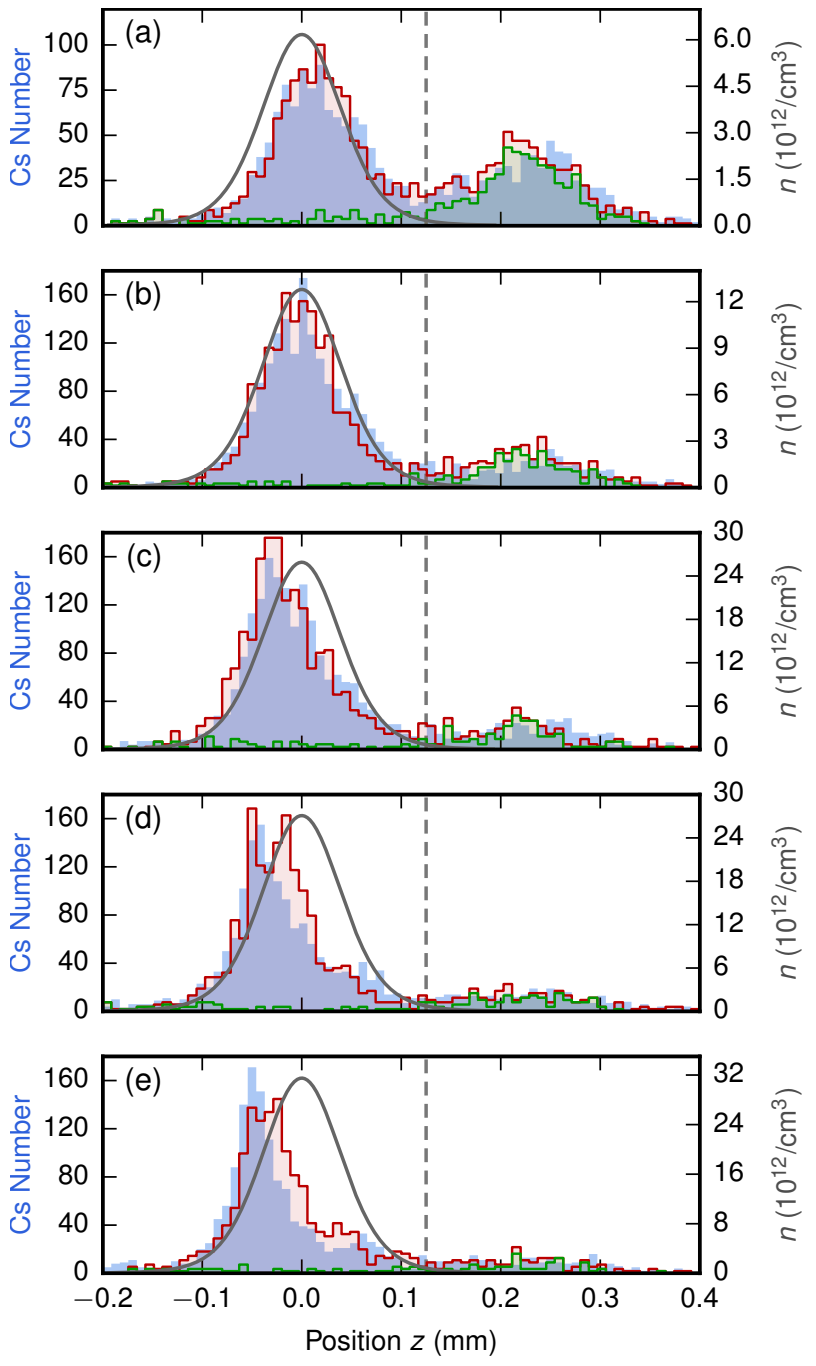

FIG. 7. Measured Cs distributions (blue histograms, borderless) for various peak $\mathrm{Rb}$ densities at $t=25 \mathrm{~ms}$ with collision simulated distributions (red histogram, solid border), normalized to the diffused fraction. In green (solid border), the fraction of atoms that have not underwent any collision are shown.

and unit variance, and 21]

$$
\begin{aligned}
\psi^{2}(v) & =2 m e^{\frac{m v^{2}}{k T}} \int_{v}^{\infty}-\gamma(u) u e^{-\frac{m u^{2}}{k T}} d u \\
& =2^{\frac{5}{2}} n \sigma \sqrt{\frac{M k T}{\pi}} \frac{M m v^{2}+(M+10 m) k T}{15(M+m)} .
\end{aligned}
$$

Equation (11) corresponds to multiplicative noise 26. On the other hand, for the usual Langevin equation the friction coefficient is velocity independent and reads [13, 27,

$$
\gamma=n \sigma \frac{8}{3} \sqrt{\frac{2 M k T}{\pi}} \frac{m}{(m+M)}
$$
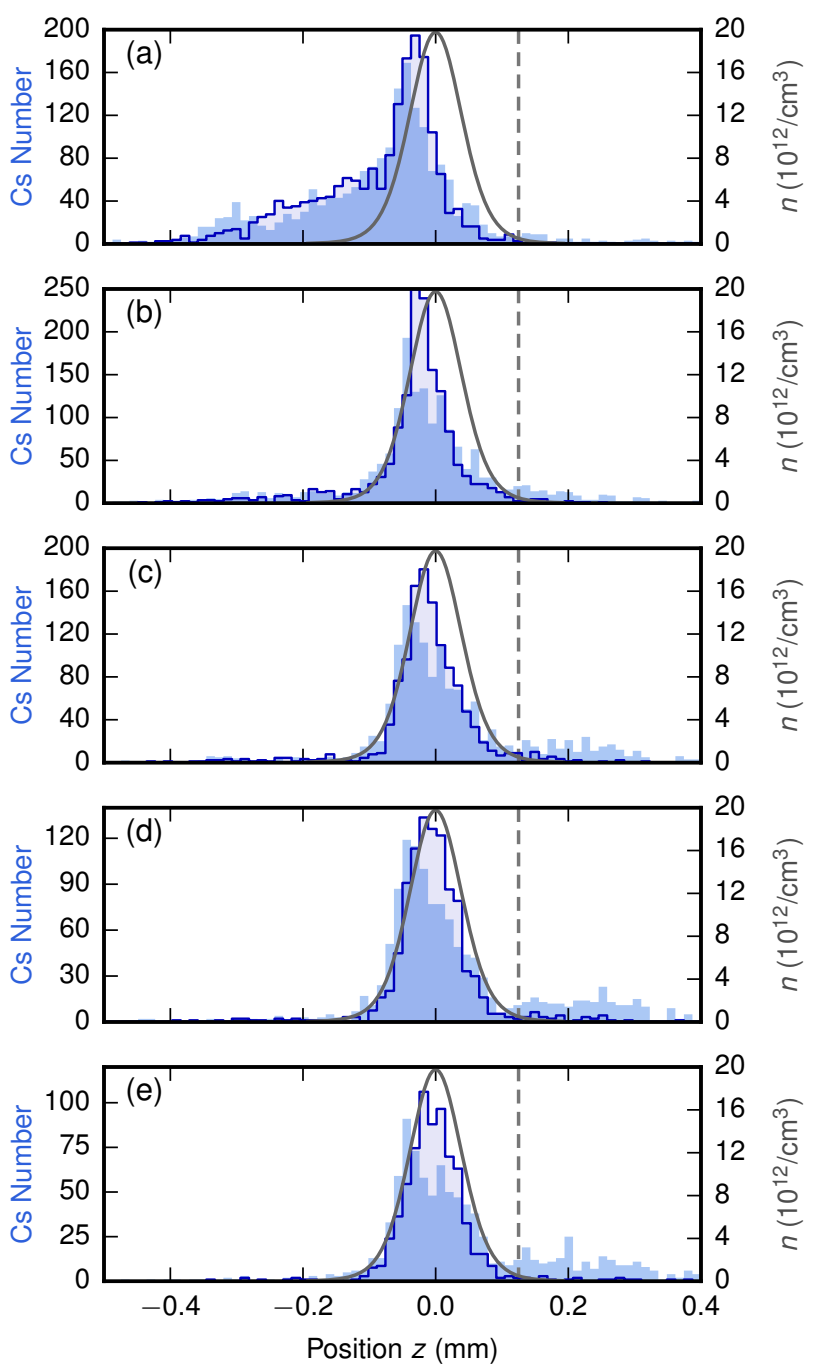

FIG. 8. Measured Cs distributions (blue histograms, borderless) for a fixed $\mathrm{Rb}$ density and growing interaction time $t=17,21,25,29,33 \mathrm{~ms}$ from (a)-(e) with Langevin simulated distributions (blue histogram, solid border), normalized to the diffused fraction.

The random force is accordingly characterized by

$$
\psi^{2}=m \gamma k T
$$

Equation (13) corresponds to additive noise [26].

\section{F. Simulation parameters}

Our numerical simulations are conducted without free parameters. The simulation parameters are obtained from independent measurements. The trapping potential consists of two Gaussian beams at a wavelength of $\lambda=1064 \mathrm{~nm}$ and the potential is precisely known from beam profile measurements, consistent with trapfrequency measurements with $\mathrm{Rb}$ and measured single $\mathrm{Cs}$ 


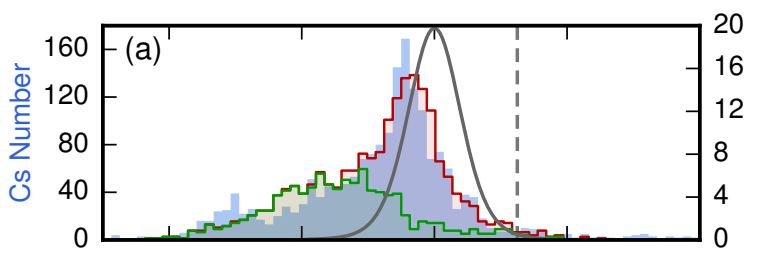

\begin{tabular}{|c|}
\hline $\bar{v}$ \\
\hline
\end{tabular}

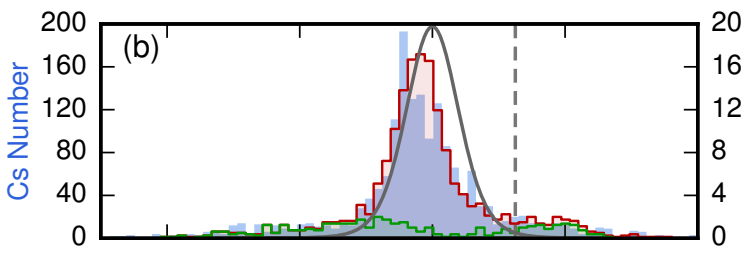

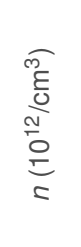

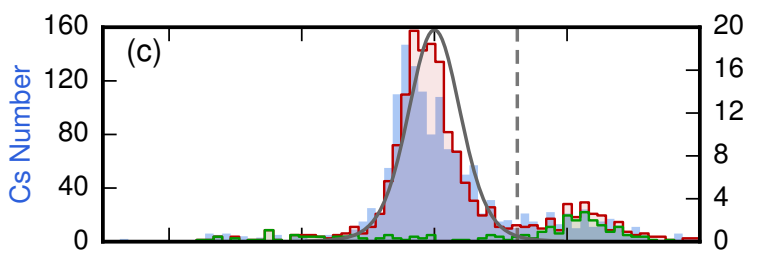

$\frac{\text { कृ }}{\frac{\text { ले }}{\text { N }}}$

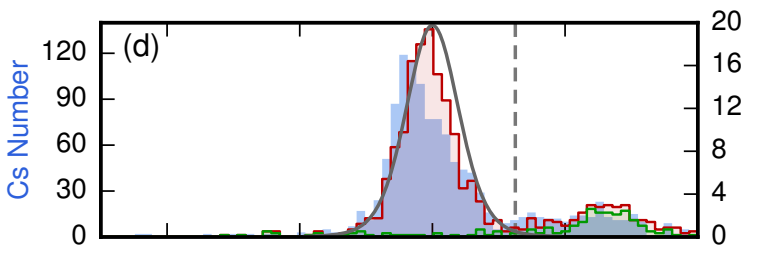

लำ

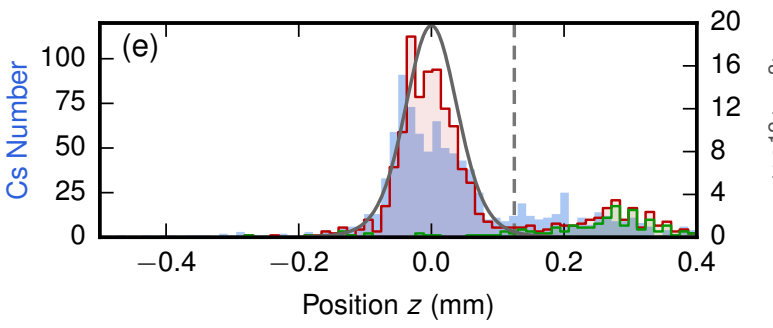

FIG. 9. Measured Cs distributions (blue histograms, borderless) for a fixed $\mathrm{Rb}$ density and growing interaction time $t=17,21,25,29,33 \mathrm{~ms}$ from (a)-(e) with collision simulated distributions (red histogram, solid border), normalized to the diffused fraction. In green (solid border), the fraction of atoms that have not underwent any collision are shown.

atom motion in absence of Rb. The vertical beam along the $x$ axis has a power of $4 \mathrm{~W}$. Its focus is located at $x=4 \mathrm{~cm}$ and its beam waist is $0.16 \mathrm{~mm}$, where the intersection of the two beams defines the coordinate system's origin. The horizontal beam along $z$ is elliptical with beam waists of $w_{x}=21 \mu \mathrm{m}$ and $w_{y}=22 \mu \mathrm{m}$, a power of $680 \mathrm{~mW}$, and its focus at $z=0$. The beams produce a potential per intensity $I$ of $U / I=-3.60 \times 10^{-36} \mathrm{Jm}^{2} / \mathrm{W}$ for Cs and $U_{\mathrm{Rb}} / I=-2.10 \times 10^{-36} \mathrm{Jm}^{2} / \mathrm{W}$ for $\mathrm{Rb}$.

The thermal distribution of $\mathrm{Rb}$ in the trap is calculated according to Boltzmann's law

$$
n(\vec{r})=N e^{\frac{-U_{\mathrm{Rb}}(\vec{r})}{k T}}\left(\int e^{\frac{-U_{\mathrm{Rb}}(\vec{r})}{k T}}\right)^{-1}
$$

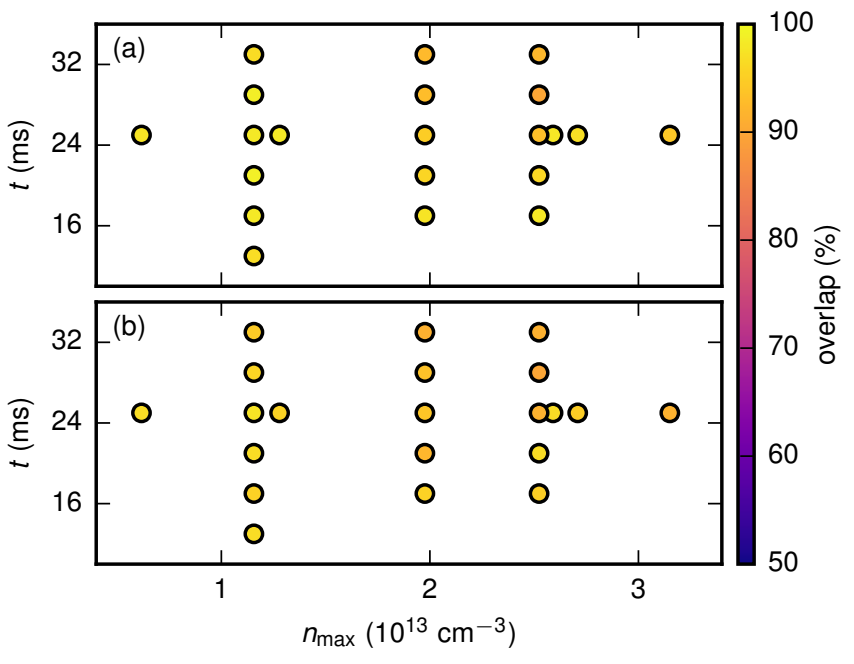

FIG. 10. Overlaps $\left(\int N_{\exp } N_{\operatorname{sim}} d z\right) /\left(\int N_{\exp }^{2} d z \int N_{\operatorname{sim}}^{2} d z\right)^{\frac{1}{2}}$ for all tracer diffusion measurements at peak densities $n_{\max }$ and interaction times $t$. Overlaps for the hard-sphere collision simulation are shown in (a), overlaps for the Langevin simulation with velocity-dependent friction, computed for the diffused fraction, are shown in (b). All overlaps for both models are $\geq 90 \%$, whereas the Langevin simulation with velocityindependent friction shown in Fig. 4 of the main article yields overlaps down to $60 \%$.

while $T$ and the Rb atom number $N$ are determined with time-of-flight thermometry and absorption imaging [28. It is important to note that a harmonic approximation of the trapping potential does not hold. We consider instead the exact Gaussian beam potential throughout the simulation. The interspecies scattering length $a_{\mathrm{Rb}, \mathrm{Cs}}=645 a_{B}$ 22] for $\mathrm{Rb}$ in $\left|F=1, m_{F}=1\right\rangle$ and $\mathrm{Cs}$ in $\left|F=3, m_{F}=3\right\rangle$ is used as an approximation for the different hyperfine states employed in this experiment.

\section{G. Detailed density distribution measurements}

The full measurement of the Cs spatial distribution when the $\mathrm{Rb}$ density is varied at a fixed interaction time corresponding to Figs. 2, 4 is shown in Figs. 6,77, together with Langevin and discrete hard-sphere collision simulations. Spatial distributions for various interaction times at a fixed Rb density, corresponding to Fig. 1, are shown in Figs. 8 9. A comparison of the overlaps for a full, consistent measurement of different densities and interaction times is depicted in Fig. 10, where the overlap of measured data with both the collision simulation (Fig. 10(a)) and the Langevin model with velocity-dependent friction coefficient (Fig. 10.(b)) yields values $\geq 90 \%$. By contrast, a Langevin model with velocity-independent friction coefficient yields typical overlaps of $\approx 60 \%$. We emphasize that the simulations have been performed with a single, consistent set of independently measured parameters for all data, without free parameter. 
[1] G. A. Bird, Molecular Gas Dynamics and the Direct Simulation of Gas Flows, (Oxford University Press, 1994).

[2] C. Cercignani, Rarified Gas Dynamics, (Cambridge University Press, 2000).

[3] E. P. Muntz, Ann. Rev. Fluid Mech. 21, 387 (1989).

[4] F. Sharipov, Rarefied Gas Dynamics: Fundamentals for Research and Practice (Wiley, 2016).

[5] A. J. Masters and T. Keyes, Phys. Rev. A 27, 2603 (1983).

[6] W. Coffey, Y. Kalmykov, and J. Waldron, The Langevin Equation (World Scientific, 1996).

[7] D. Gillespie and E. Seitaridou, Simple Brownian Diffusion (Oxford University Press, 2012).

[8] P. Mazur and I. Oppenheim, Physica 50, 241 (1970).

[9] J. Blum, S. Bruns, D. Rademacher, A.Voss, B. Willenberg, and M. Krause, Phys. Rev. Lett. 97, 230601 (2006).

[10] T. Li, S. Kheifets, D. Medellin, and M.G. Raizen, Science 328, 1673 (2010).

[11] R. Huang, I. Chavez, K. M. Taute, B. Lukic, S. Jeney, M. G. Raizen, and E.-L. Florin, Nature Phys. 7, 576 (2011).

[12] S. Kheifets, A. Simha, K. Melin, T. Li, and M. G. Raizen, Science 343, 1493 (2014).

[13] L. Ferrari, Chem. Phys. 336, 27 (2007).

[14] L. Ferrari, Chem. Phys. 428, 144 (2014).

[15] H. J. Metcalf and P. van der Straten, Laser Cooling and Trapping (Springer, 2002).

[16] R. Mazo, Brownian Motion (Oxford University Press, 2002).
[17] M. Hohmann, F. Kindermann, B. Gänger, T. Lausch, D. Mayer, F. Schmidt, and A. Widera, EPJ Quantum Technology 2, 1 (2015).

[18] M. Hohmann, F. Kindermann, T. Lausch, D. Mayer, F. Schmidt, and A. Widera, Phys. Rev. A 93, 043607 (2016).

[19] See Supplementary Material for details.

[20] S. Chapman and T. Cowling, The Mathematical Theory of Non-uniform Gases (Cambridge University Press, 1970).

[21] A. A. Dubkov, P. Hänggi, and I. Goychuk, J. Stat. Mech. P01034 (2009).

[22] T. Takekoshi, M. Debatin, R. Rameshan, F. Ferlaino, R. Grimm, H.-C. Nägerl, C. R. Le Sueur, J. M. Hutson, P. S. Julienne, S. Kotochigova, E. Tiemann, Phys. Rev. A 85, 032506 (2012).

[23] C.J. Pethik and H. Smith, Bose-Einstein Condensation in Dilutes Gases (Cambridge University Press, 2002).

[24] E. G. M. van Kempen, S. J. J. M. F. Kokkelmans, D. J. Heinzen, and B. J. Verhaar, Phys. Rev. Lett. 88, 093201 (2002).

[25] N. Spethmann, F. Kindermann, S. John, C. Weber, D. Meschede, and A. Widera, Phys. Rev. Lett. 109, 235301 (2012)

[26] J. M. Sancho, M. San Miguel, S. L. Katz, and J. D. Gunton, Phys. Rev. A 26, 1589 (1982).

[27] L. Ferrari, Chem. Phys. 257, 63 (2000).

[28] Inguscio, M., Stringari, S., Wieman, C.E.,Proceedings of the International School of Physics "Enrico Fermi", Course CXL (1998). 